Aarão Mendes Pinto Neto ${ }^{1}$

Adriana Orcesi Pedro 1

Ellen Hardy 2

Maria José Duarte Osis 2

Lúcia Helena Simões da Costa-Paiva 1

Edson Zangiacomi Martinez ${ }^{1}$

\section{Caracterização das usuárias de terapia de reposição hormonal do Município de Campinas, São Paulo}

\author{
Characterization of hormone replacement \\ therapy users in Campinas, São Paulo
}

1 Departamento de Tocoginecologia, Faculdade de Ciências Médicas, Universidade Estadual de Campinas. C. P. 6181, Campinas, $S P$ 13081-970, Brasil.

aarao@obelix.unicamp.br 2 Departamento de Pesquisas Médico-Sociais, Centro de Pesquisas das Doenças Materno-Infantis de Campinas. C. P. 6181 , Campinas, $S P$ 13081-970, Brasil. hardy@unicamp.br

\begin{abstract}
This study employed a descriptive, cross-sectional, population-based design to characterize climacteric women from Campinas, São Paulo State, based on use of hormone replacement therapy (HRT). An area cluster sample was selected with 456 women 45 to 60 years of age, residing in Campinas, based on data from the Brazilian Institute of Statistics and Geography (IBGE). Women were selected by area cluster, and the reference unit was the census tract as defined by the IBGE. Data were collected through home interviews using a structured and pre-tested questionnaire provided by the International Health Foundation/International Menopause Society and by the North American Menopause Society and adapted by the authors. In order to characterize women according to current, past, or no use of HRT, a polytonic logistic regression model was used, with a backward selection process of variables. The authors conclude that the main characteristics of HRT users in the city of Campinas were perimenopausal status and higher literacy and socioeconomic class.
\end{abstract}

Key words Menopause; Hormone Replacement Therapy; Women's Health

Resumo Realizou-se um estudo descritivo de corte transversal, de base populacional, com o objetivo de caracterizar as mulheres climatéricas do Município de Campinas, Estado de São Paulo, de acordo com o uso da terapia de reposição hormonal (TRH). Selecionaram-se, por processo de amostragem, 456 mulheres na faixa etária de 45-60 anos de idade, segundo informações obtidas da agência local da Fundação Instituto Brasileiro de Geografia e Estatística (IBGE). As mulheres foram selecionadas por conglomerados e a unidade de referência foi um setor censitário, conforme definido pelo IBGE. Os dados foram coletados por intermédio de entrevistas domiciliares, com questionários estruturados e pré-testados, fornecidos pela Fundação Internacional de Saúde, Sociedade Internacional de Menopausa e pela Sociedade Norte-Americana de Menopausa e adaptados pelos autores. Para caracterizar as mulheres segundo o uso atual ou passado de TRH, utilizou-se o modelo de regressão logística politômica, com processo de seleção backward de variáveis. Concluiu-se que no Município de Campinas as principais características das usuárias da terapia de reposição hormonal foram estar na perimenopausa, ter maior escolaridade e melhor classe social.

Palavras-chave Menopausa; Terapia de Reposição Hormonal; Saúde da Mulher 


\section{Introdução}

Como clínico, quer atuando na atenção primária, secundária ou terciária, necessita-se de conhecer a mulher que procura atendimento e, no caso específico das mulheres climatéricas, caracterizar as usuárias da terapia de reposição hormonal (TRH). Whitehead (1994) relatou que o grau de ansiedade, irritabilidade e depressão são os principais fatores que diferenciam mulheres que buscam atendimento especializado e terapia de reposição hormonal das que não procuram atendimento ou tratamento.

As usuárias têm um estilo de vida mais saudável, melhor classe social, melhores salários e melhor nível educacional, assim como são grandes usuárias de serviços médicos preventivos para procedimentos como a mamografia, citologia oncológica e avaliação dos níveis de colesterol. Outro determinante importante para o uso da terapia de reposição hormonal é a presença de sintomas climatéricos, especificamente as ondas de calor, para as quais a terapia hormonal é geralmente prescrita. Estudos também têm demonstrado diferenças raciais no uso de TRH, sendo as brancas as maiores usuárias. A raça também tem sido relacionada à classe sócio-econômica e à utilização de serviços de saúde (Avis \& Johanes, 1998; Stafford et al., 1998).

A decisão de uma mulher em usar terapia de reposição hormonal é um processo complexo determinado pela recomendação de seu médico, pelo risco individual de doenças, pelas atitudes frente à menopausa e TRH, valores, sintomas menopausais e ao meio ao qual a mulher pertence. Precisávamos caracterizar as usuárias de TRH em nosso meio e, com este objetivo, realizou-se este estudo, o primeiro de base populacional com um grupo de mulheres brasileiras.

\section{Casuística e métodos}

Tratou-se de um estudo descritivo de corte transversal, tipo inquérito populacional domiciliar. O tamanho da população-alvo considerado para este cálculo foi a população feminina de Campinas, São Paulo, na faixa etária entre 45 a 60 anos, no ano de 1997. Este dado foi obtido por uma projeção por meio de regressão linear da população, baseada no número de mulheres nesta faixa etária residentes no Município de Campinas no último Censo Demográfico (IBGE, 1994), e com projeção de população estimada para o ano de 1997, sendo este total de 79.727. Tal procedimento foi reali- zado pelo Laboratório Aplicado de Epidemiologia do Departamento de Medicina Social da Universidade Estadual de Campinas, utilizando-se a regressão linear da população, baseando-se em método descrito por Laurenti et al. (1987). Desta forma, o número mínimo calculado de entrevistas foi de 367, mas foi acrescido de $20 \%$ para evitar possíveis perdas durante o estudo. Selecionaram-se, por intermédio de processo de amostragem, 456 mulheres na faixa etária de 45-60 anos de idade, segundo informações obtidas da agência local da Fundação Instituto Brasileiro de Geografia e Estatística (IBGE). As mulheres foram selecionadas por conglomerados e a unidade de referência foi um setor censitário, conforme definido pelo IBGE (1994). Estudaram-se 82 setores censitários que foram sorteados entre todos os setores censitários de Campinas. Em cada um dos setores censitários selecionaram-se no mínimo uma e no máximo seis mulheres, para as quais uma entrevistadora treinada aplicava um checklist para evitar possíveis erros de seleção.

A coleta dos dados foi realizada por quatro entrevistadoras e uma supervisora que receberam $32 \mathrm{~h}$ de treinamento, com aulas teóricas e práticas, incluindo realização de entrevistas simuladas e reais. Para assegurar a qualidade dos dados coletados, foram realizadas medidas de controle de qualidade que incluíram, além do treinamento das entrevistadoras e supervisora, a preparação e uso do manual de instrução para as entrevistadoras, monitorização da coleta, checagem durante trabalho de campo, checagem do preenchimento completo e fidedignidade da entrevista pela supervisora e pesquisador principal.

Utilizou-se um questionário estruturado e pré-testado para obter as informações de interesse para o estudo. Todas as variáveis basearam-se no auto-relato das mulheres. Tal questionário foi elaborado com base em dois questionários adaptados pelos autores e por membros do Departamento de Pesquisas MédicoSociais do Centro Materno Infantil de Campinas (CEMICAMP). O primeiro questionário foi fornecido pela Sociedade Internacional de Menopausa e Fundação Internacional de Saúde e aplicado em sete países do Sudeste Asiático em 1993 (Boulet et al., 1994). O segundo foi fornecido pela Sociedade Norte-Americana de Menopausa e aplicado nos Estados Unidos (Utian \& Schiff, 1994). De um modo geral, a receptividade das mulheres abordadas em responder às perguntas foi boa e a proporção de recusas foi de $11,2 \%$. A metodologia, em detalhes, foi descrita em estudos anteriores (Pedro, 1999; Pinto Neto, 2000). 
As variáveis estudadas foram a idade; idade à menopausa (tempo decorrido desde a última menstruação natural e expresso em anos, meses ou dias); cor; tabagismo; estado marital; nível educacional; emprego; classe sócio-econômica segundo critérios da Associação Brasileira de Anunciantes (ABA) e da Associação Brasileira dos Institutos de Pesquisa de Mercado (ABIPEME), classificado de acordo com a pontuação de Almeida \& Wickerhauser (1991); estado menopausal (segundo definição de Jaszmann, 1973: pré-menopausa - mulheres com ciclos menstruais regulares ou com padrão menstrual similar ao que elas tiveram durante a vida reprodutiva; perimenopausa - ciclos menstruais regulares nos últimos 12 meses, mas com mudança do padrão menstrual; pósmenopausa - último período menstrual ocorreu há pelo menos 12 meses antes da entrevista); uso de terapia de reposição hormonal; tipo de menopausa (natural ou cirúrgica); religião; coabitação com filhos e netos. Para buscar um grupo destas variáveis que melhor caracterizasse a população segundo o uso atual $(n=88)$, uso no passado $(n=84)$ e as que nunca utilizaram TRH $(n=284)$, utilizou-se um modelo de regressão logística politômica, com processo de seleção backward de variáveis. Tal critério consistiu em ajustar um modelo inicial, considerando todas as variáveis que, na análise bivariada, associavam-se ao de uso de TRH com valor de p menor ou igual a 0,10. Tendo este modelo como fundamento, foram eliminadas, uma a uma, as variáveis que passaram a não ter evidências de caracterizar, naquele contexto, a situação de uso de TRH com nível de significância de 0,05. Ajustou-se um novo modelo a cada variável eliminada, e o processo se repetiu até que fosse encontrado um modelo que considerasse apenas variáveis que efetivamente caracterizassem a situação de uso de TRH. O programa utilizado para esses procedimentos estatísticos foi o SAS, versão 6.12 (SAS Institute Incoporation, 1996).

Foi redigido um Termo de Consentimento Livre e Esclarecido, conforme as Normas do Conselho Nacional de Saúde, explicitadas na Resolução 196/96 (CNS, 1996). Lia-se este Termo a cada mulher identificada como possível participante do estudo, quando convidada a participar do mesmo.

\section{Resultados}

A Tabela 1 mostra as características que se associaram ao uso de terapia de reposição hormonal na análise bivariada.
A Tabela 2 refere-se às características associadas ao uso da terapia de reposição hormonal, em modelo de análise múltipla. Observouse que as mulheres na perimenopausa apresentaram uma chance 4,03 vezes maior de já ter utilizado TRH em relação às mulheres que se encontravam na pré-menopausa. Essas mulheres também apresentaram uma chance quase três vezes maior em relação ao grupo de referência, quando comparadas usuárias e não usuárias de TRH.

A maior escolaridade (segundo grau e universitário) aumentou quatro vezes a chance de a mulher estar em uso atual de terapia de reposição hormonal, em relação às que nunca utilizaram tal tratamento. As classes sociais mais elevadas relacionaram-se aos maiores valores de odds ratio (OR), tanto em ex-usuárias $(4,72)$ como em usuárias atuais $(6,87)$ de terapia hormonal, em relação às mulheres que nunca utilizaram tal tratamento.

As mulheres que não declaram o hábito de fumar apresentaram chance três vezes maior (OR de 3,2) de já terem utilizado terapia de reposição hormonal em relação às que nunca a utilizaram.

\section{Discussão}

A caracterização das mulheres, segundo o uso ou não de TRH, mostrou diferenças significativas entre os grupos. O maior percentual de usuárias e ex-usuárias concentrou-se nas faixas etárias superiores. O mesmo padrão, como esperado, ocorreu em relação ao estado menopausal, pois este associou-se fortemente à faixa etária, ou seja, usuárias e ex-usuárias foram predominantemente mulheres na peri e pósmenopausa. Também ficou evidente que mulheres de maior escolaridade ou estavam utilizando ou já utilizaram TRH. O tabagismo predominou entre as não usuárias, e as mulheres brancas foram maioria entre as usuárias e exusuárias da terapia hormonal, provavelmente sugerindo associação entre cor e classe sócioeconômica. A influência dos filhos coabitando na mesma moradia também mostrou diferenças significativas entre os grupos, com maior percentual entre usuárias e ex-usuárias. As classes sócio-econômicas mais elevadas predominaram entre usuárias e ex-usuárias de TRH.

A comparação de usuárias e não usuárias de TRH tem mostrado que, em geral, um fator importante associado ao uso é a ocorrência de menopausa cirúrgica. Porém, o estilo de vida saudável, classes sócio-econômicas elevadas, 
Características que significativamente se associaram ao uso de terapia de reposição hormonal. Análise bivariada.

\begin{tabular}{|c|c|c|c|c|c|c|c|}
\hline & \multicolumn{6}{|c|}{ Uso de TRH } & \multirow[t]{2}{*}{$\mathbf{p}$} \\
\hline & n & $\%$ & $\mathrm{n}$ & $\%$ & $\mathrm{n}$ & $\%$ & \\
\hline \multicolumn{8}{|l|}{ Faixa etária } \\
\hline 45 a 49 & 121 & 70,4 & 30 & 17,4 & 21 & 12,2 & $<0,01(1)$ \\
\hline 50 a 54 & 80 & 55,6 & 37 & 25,7 & 27 & 18,7 & \\
\hline 55 a 60 & 83 & 59,3 & 21 & 15,0 & 36 & 25,7 & \\
\hline \multicolumn{8}{|l|}{ Estado menopausal } \\
\hline Pré-menopausa & 89 & 76,1 & 16 & 13,7 & 12 & 10,3 & $<0,01(1)$ \\
\hline Perimenopausa & 56 & 70,0 & 12 & 15,0 & 12 & 15,0 & \\
\hline Pós-menopausa & 139 & 53,9 & 59 & 22,9 & 60 & 23,2 & \\
\hline \multicolumn{8}{|l|}{ Escolaridade } \\
\hline Sem & 59 & 81,9 & 4 & 5,6 & 9 & 12,5 & $<0,01(1)$ \\
\hline 1ạ a 4ạ série do 1o grau & 145 & 59,7 & 48 & 19,7 & 50 & 20,6 & \\
\hline 5a a $8 \underline{a}$ série do 1 o grau & 55 & 67,9 & 16 & 19,7 & 10 & 12,4 & \\
\hline 2o grau e universidade & 25 & 41,7 & 20 & 33,3 & 15 & 25,0 & \\
\hline \multicolumn{8}{|l|}{ Tabagismo } \\
\hline Fuma atualmente & 65 & 69,9 & 21 & 22,6 & 7 & 7,5 & $0,01(1)$ \\
\hline Não fuma atualmente & 219 & 60,3 & 67 & 18,5 & 77 & 21,2 & \\
\hline \multicolumn{8}{|l|}{ Cor declarada } \\
\hline Branca & 139 & 54,1 & 63 & 24,5 & 55 & 21,4 & $<0,01(1)$ \\
\hline Parda/mulata/preta & 93 & 74,4 & 16 & 12,8 & 16 & 12,8 & \\
\hline Outra & 52 & 70,3 & 9 & 12,2 & 13 & 17,6 & \\
\hline Não tem filhos morando junto & 75 & 62,5 & 23 & 19,2 & 22 & 18,3 & $0,03(1)$ \\
\hline Tem um filho morando junto & 64 & 51,6 & 34 & 27,4 & 26 & 21,0 & \\
\hline Tem 2 ou mais filhos morando junto & 145 & 68,4 & 31 & 14,6 & 36 & 17,0 & \\
\hline \multicolumn{8}{|l|}{ Naturalidade } \\
\hline Campinas & 40 & 50,0 & 20 & 25,0 & 20 & 25,0 & $0,06(1)$ \\
\hline Outra cidade de São Paulo & 134 & 61,4 & 42 & 19,3 & 42 & 19,3 & \\
\hline Outra cidade & 110 & 69,6 & 26 & 16,5 & 22 & 13,9 & \\
\hline \multicolumn{8}{|l|}{ Classe $\left(^{*}\right)$} \\
\hline$A$ e $B$ & 35 & 38,5 & 32 & 35,2 & 24 & 26,4 & $<0,01(1)$ \\
\hline C & 104 & 56,8 & 40 & 21,9 & 39 & 21,3 & \\
\hline$D$ e $E$ & 144 & 79,6 & 16 & 8,8 & 21 & 11,6 & \\
\hline
\end{tabular}

(1) Teste qui-quadrado de Pearson

(*) Falta uma informação

TRH = terapia de reposição hormonal

maior escolaridade e o uso de procedimentos preventivos de saúde também influenciam significativamente a utilização de hormônios (Avis \& Johanes, 1998). Outros fatores importantes são a presença da sintomatologia climatérica (Johanes et al., 1994), sendo o uso mais evidente entre mulheres brancas (Dawson \& Tompson, 1990; Egland et al., 1988).
Nesta população, as características que se associaram significativamente ao uso de TRH, observadas mediante análise multivariada, foram o estado menopausal, a escolaridade e a classe social. O uso de um modelo de regressão logística se justifica pela natureza do estudo considerar apenas variáveis categóricas, o que impossibilita métodos mais adequados ao pro- 
Características que significativamente se associaram ao uso de terapia de reposição hormonal. Análise multivariada.

\begin{tabular}{|c|c|c|c|c|c|c|}
\hline & \multicolumn{3}{|c|}{ Já usou/nunca usou } & \multicolumn{3}{|c|}{ Usa atualmente/nunca usou } \\
\hline & Odds Ratio & IC $95 \% \mathrm{LI}$ & IC $95 \%$ LS & Odds Ratio & IC 95\% LI & IC $95 \%$ LS \\
\hline \multicolumn{7}{|l|}{ Estado menopausal } \\
\hline Pré-menopausa & referência & - & - & referência & - & - \\
\hline Perimenopausa & 4,03 & 1,97 & 8,22 & 2,95 & 1,52 & 5,73 \\
\hline Pós-menopausa & 2,02 & 0,81 & 5,03 & 1,51 & 0,63 & 3,60 \\
\hline \multicolumn{7}{|l|}{ Escolaridade } \\
\hline Sem & referência & - & - & referência & - & - \\
\hline 1ạ a 4a série do 1o grau & 1,38 & 0,60 & 3,15 & 2,96 & 0,98 & 8,91 \\
\hline 5a a 8 a série do 1 o grau & 0,69 & 0,24 & 2,01 & 2,31 & 0,68 & 7,89 \\
\hline 2o grau e universidade & 1,71 & 0,55 & 5,26 & 4,00 & 1,08 & 14,83 \\
\hline \multicolumn{7}{|l|}{ Classe social } \\
\hline$D$ e $E$ & referência & - & - & referência & - & - \\
\hline C & 2,59 & 1,37 & 4,90 & 3,01 & 1,54 & 5,90 \\
\hline$A$ e $B$ & 4,72 & 2,04 & 10,96 & 6,87 & 2,99 & 15,79 \\
\hline \multicolumn{7}{|l|}{ Fumo } \\
\hline Fuma atualmente & referência & - & - & referência & - & - \\
\hline Não fuma atualmente & 3,29 & 1,40 & 7,70 & 0,87 & 0,47 & 1,62 \\
\hline
\end{tabular}

IC = Intervalo de confiança; LI = Limite inferior; $L S=$ Limite superior

blema de caracterizar populações segundo variáveis, como por exemplo, a análise discriminante. Este método tem uma teoria baseada em variáveis contínuas, e, segundo Johnson \& Wichern (1982), dentre as alternativas apresentadas pela literatura estatística, uma das mais adequadas seria o uso de modelos de regressão logística.

Mulheres na perimenopausa apresentaram o dobro de probabilidade de estarem usando e quatro vezes maior de já terem utilizado TRH, tendo como referência mulheres na pré-menopausa. Este resultado pode ser explicado pela elevada prevalência de sintomas climatéricos na população, visto que $96,9 \%$ das mulheres experimentaram pelo menos um dos sintomas, sendo as ondas de calor os mais freqüentes, com prevalência maior em mulheres na perimenopausa (Pedro, 1999). Pinto Neto (2000) constatou que a presença dos sintomas climatéricos foi considerada uma desvantagem significativa durante a transição climatérica. A análise multivariada também evidenciou probabilidade muito maior de uso de TRH em mulheres de maior escolaridade e principalmente de classe social elevada (OR 4,0 e 6,87, respectivamente).

Em 1997, Brett \& Madans avaliaram as diferenças raciais relacionadas ao uso de TRH em uma amostra representativa de mulheres americanas, controladas pela classe sócio-econômica. Relataram uma alta probabilidade de uso entre mulheres brancas de maior escolaridade. As mulheres brancas apresentaram quase o dobro de probabilidade que as negras. Esta diferença não se modificou após o controle de outras variáveis. Quando estratificadas por educação, as mulheres brancas permaneceram as maiores usuárias de TRH em todos os níveis educacionais. Estes resultados sugerem que a raça foi uma variável independente relacionada ao uso de TRH. Nesta casuística, apesar de a cor declarada ter apresentado diferença significativa à análise bivariada, esta relação não permaneceu quando realizamos a análise multivariada. Tal fato pode se dever a maior relação, em nosso meio, entre escolaridade, classe sócio-econômica e cor da pele.

Sttafford et al. (1998) avaliaram os dados de visitas médicas de mulheres americanas com 40 anos ou mais, no período de 1989 e 1996, e observaram maior uso de TRH em mulheres com sintomas. Naquelas sem sintomatologia exuberante, as que consultaram tocoginecologistas apresentaram maior prevalência de uso de terapia hormonal. Relataram também que a cor branca e os maiores salários associaram-se ao uso de hormônios durante o climatério. Es- 
tas diferenças diminuíram ao longo do tempo e, em 1995-1996, somente a raça permaneceu como uma variável independente. Concluíram que apesar destas diferenças, as influências de outras variáveis permaneceram. Este estudo avaliou somente mulheres que procuraram atenção médica. Relataram também que as usuárias procuraram serviços médicos predominantemente para prevenção e que as atendidas na região ocidental dos Estados Unidos receberam mais prescrições que as de outras regiões do país.

Neste estudo, as mulheres que já utilizaram TRH apresentaram chance de não fumar três vezes maior. É provável que o contato com serviços de saúde tenha propiciado maiores informações sobre estilo de vida e, especialmente, sobre hábitos deletérios para a saúde como o hábito de fumar. Conforme dados de estudo realizado anteriormente, a prevalência de uso de TRH nesta população foi de 19\% (Pedro, 1999), muito superior às taxas relatadas em países sul-americanos cuja prevalência foi de 2,8\% em 1990, 5,6\% em 1996, com estimativa de $10 \%$ no ano 2000 (Armendáriz, 1998). Segundo Sttafford et al. (1998), as diferenças regionais existem mesmo em países desenvolvidos como os Estados Unidos da América. Portanto, podemos inferir que os dados deste estudo não são aplicáveis a outras regiões do Brasil, um país de dimensões continentais, com padrão cultural diferente em cada uma de suas regiões. As diferenças também são marcantes em relação aos aspectos sociais, econômicos e demográficos. Deste modo, seria preferível que houvéssemos incluído nesta casuística somente mulheres nascidas em Campinas, porém isto dificultaria e, particularmente, elevaria muito os custos da pesquisa.

\section{Agradecimentos}

À Fundação de Amparo à Pesquisa do Estado de São Paulo e Fundo de Apoio ao Ensino e à Pesquisa, da Universidade Estadual de Campinas.
As influências do estado menopausal, da escolaridade e da classe social no uso da terapia hormonal reafirmaram observações anteriores nesta mesma população. Pedro (1999) relatou que as mulheres de classes sócio-econômicas menos favorecidas, com baixa escolaridade e com sintomas climatéricos intensos, apresentaram autopercepção negativa do estado de saúde, evidenciando a injustiça com que a sociedade como um todo, e notadamente os sistemas social e de saúde vigentes, segrega as mulheres climatéricas de classes sociais menos favorecidas. Além das dificuldades de ser uma mulher de meia-idade numa sociedade como a atual, a exclusão por fator econômico e social é uma realidade. Esta exclusão é um fenômeno quase universal, ou seja, não é exclusivo desta região e desta população.

Mais ainda, Shelley et al. (1995) relataram que a observação das populações incluídas nos grandes estudos observacionais sugere existirem diferenças substanciais entre usuárias e não usuárias de terapia de reposição hormonal, fazendo com que a avaliação dos resultados do TRH pudesse ser, ao menos em parte, devida mais ao viés de seleção do que à terapia propriamente dita.

Finalmente, torna-se importante salientar que o interesse em terapia de reposição hormonal tem crescido muito nos últimos anos, mesmo em países em desenvolvimento, como o Brasil. Por ser o primeiro estudo nacional dessa natureza, acredita-se que os resultados desta pesquisa possam, de alguma maneira, racionalizar o uso da terapia de reposição hormonal em nosso meio e, conseqüentemente, melhorar a sua aceitação, além de oferecer um atendimento direcionado às necessidades das mulheres de meia-idade.

\section{Referências}

ALMEIDA, P. M. \& WICKERHAUSER, H., 1991. O Critério ABA/ABIPEME - Em Busca de uma Atualização. São Paulo: Associação Brasileira de Anunciantes/Associação Brasileira dos Institutos de Pesquisas de Mercado. (mimeo.)

ARMENDARIZ, M. P., 1998. Realidad demográfica em Latinoamérica y tratamiento hormonal de reemplazo. In: Menopausia y Longevidad: Perspectiva Clínica e Epidemológica em Latinoamérica (O. G. Campos, E. A. Urzua \& P. C. Castro, ed.), pp. 7388, Santiago de Chile: Bywaters. 
AVIS, N. E. \& JOHANES, C., 1998. Socioeconomic status and HRT use. Menopause, 5:137-139.

BOULET, M. J.; ODDENS, B. J.; LEHERT, P.; VEMER, H. M. \& VISSER, A., 1994. Climacteric and menopause in seven South-east Asian countries. Maturitas, 19:157-176.

CNS (Conselho Nacional de Saúde), 1996. Resolução oo 196/96. Informe Epidemiológico do SUS, oo 2, Brasília: CNS.

BRETT, K. M. \& MADDANS, J. H., 1997. Differences in use of postmenopausal hormone replacement therapy by black and white women. Menopause, 4:66-70.

DAWSON, D. A. \& TOMPSON, G. B., 1990. Breast cancer risk factors and screening: Unite States, 1987. National Center for Health Statistics. Vital and Health Statistics, 172:1-60.

EGLAND, G. M.; MATTHEWS, K. A.; KULLER, L. H. \& KELSEY, S. F., 1988. Characteristics of non contraceptive hormone users. Preventive Medicine, 17: 403-411.

IBGE (Fundação Instituto Brasileiro de Geografia e Estatística), 1994. Recenseamento Geral da População, 1991. Rio de Janeiro: IBGE.

JASZMANN, L., 1973. Epidemiology of climateric and post-climateric complaints. In: Ageing and Estrogens. (P. A. Van Keep \& C. Lauritzen, ed.), pp. 2234, Basel: Karger.

JOHANES, C. B.; CRAWFORD, S. C.; POSNER, J. G. \& MSKINLAY, S. M., 1994. Longitudinal patterns and correlates of hormone replacement therapy use in middle-aged women. American Journal of Epidemiology, 40:439-452.

JOHNSON, R. A. \& WICHERN, D. W., 1982. Applied Multivariate Statistical Analysis. New Jersey: Prentice-Hall Inc./Englewood Cliffs.
LAURENTI, R.; MELLO-JORGE, M. L. P.; LEBRÃO, M L. \& GOTLIEB, S. L. D., 1987. População, recenseamento e estimativas. In: Estatística de Saúde (R. Laurenti, M. L. P. Mello-Jorge, M. L. Lebrão \& S. L. D. Gotlieb, org.), pp. 9-38, São Paulo: Editora Pedagógica Universitária.

PEDRO, A. O., 1999. Inquérito Populacional Domiciliar sobre o Climatério e a Menopausa em $\mathrm{Mu}$ lheres do Município de Campinas. Tese de Doutorado, Campinas: Faculdade de Ciências Médicas, Universidade Estadual de Campinas.

PINTO NETO, A. M., 2000. Atitudes de Mulheres Climatéricas do Município de Campinas Frente à Menopausa e seu Tratamento. Tese de Livre Docência, Campinas: Faculdade de Ciências Médicas, Universidade Estadual de Campinas.

SAS INSTITUTE INCOPORATION, 1996. The SAS System for Windows. Versão 6.12. Cary: SAS Institute Inc.

SHELLEY, J. M.; SMITH, A. M. A.; DUDLEY, E. \& DENNERSTEIN, L., 1995. Use of hormone replacement therapy by Melbourne women. Austriac Journal of Public Health, 19:387-392.

STTAFFORD, R. S.; SAGLAM, D.; CAUSINO, N. \& BLUMENTHAL, D., 1998. The declining impact of race and insurance status on hormone replacement therapy. Menopause, 5:140-144.

UTIAN, W. H. \& SCHIFF, I. N., 1994. Gallup survey on women's knowledge, information sources, and attitudes to menopause and hormone replacement therapy. Menopause, 1:39-48.

WHITEHEAD, M., 1994. The Pieter Van Keep memorial lecture. In: The Modern Management of the Menopause: A Perspective for the 21 st Century (G. Berg \& M. Hammar, ed.), pp. 1-13, New York: The Parthenon Publishing Group.

Recebido em 5 de junho de 2000

Versão final reapresentada em 26 de abril de 2001

Aprovado em 2 de julho de 2001 